\title{
Predicting the difference in treatment response and survival time of lung adenocarcinoma patients based on a prognostic risk model of glycolysis- related genes
}

\section{Rongchang Zhao ( $\nabla$ zhao8296@163.com )}

Taixing people hospital https://orcid.org/0000-0002-8453-9446

\section{Dan Ding}

Taixing People's Hospital

Yan Ding

Taixing People's Hospital

Rongbo Han

Taixing People's Hospital

\section{Xiujuan Wang}

Taixing People's Hospital

Chunrong Zhu

Soochow University Affiliated No 1 Peoplel's Hospital: First Affiliated Hospital of Soochow University

\section{Research}

Keywords: lung adenocarcinoma, glycolysis, prognostic, nomogram, drug sensitivity

Posted Date: October 11th, 2021

DOI: https://doi.org/10.21203/rs.3.rs-952541/v1

License: (c) (i) This work is licensed under a Creative Commons Attribution 4.0 International License.

Read Full License 


\section{Abstract \\ Background}

Multiple factors affect the survival time of patients with lung adenocarcinoma (LUAD). Specifically, the therapeutic effect of medicines and the disease recurrence probability differs among patients with the same stage of LUAD. Thus, effective prognostic predictors need to be identified.

\section{Methods}

Based on the tumor mutation burden (TMB) data obtained by TCGA, LUAD was divided into high and low groups, and the differentially expressed glycolysis-related genes between the two groups were screened out. Cox regression was used to obtain a prognostic model. A receiver operating characteristic (ROC) curve and calibration curve were generated to evaluate the nomogram that was constructed based on clinicopathological characteristics and the risk score. Two datasets (GSE68465 and GSE11969) from Gene Expression Omnibus (GEO) were used to verify the prognostic performance of the gene. Furthermore, differences in immune cell distribution, immune-related molecules and drug susceptibility were assessed for their relationship with the risk score.

\section{Results}

We confirmed a 5-gene signature (FKBP4, HMMR, B4GALT1, ERO1L, ENO1) capable of dividing patients into two risk groups. There was a significant difference in overall survival (OS) times between the highrisk group and the low-risk group $(P=1.085 \mathrm{e}-4)$, with the low-risk group having a better survival outcome. Through multivariate Cox analysis, the risk score was confirmed to be an independent prognostic factor $(\mathrm{HR}=1.289,95 \% \mathrm{Cl}=1.202-1.383, P<0.001)$, and the ROC curve and nomogram exhibited accurate prediction performance. Validation of the data obtained in the GEO database yielded similar results. Additionally, there were significant differences in cisplatin, paclitaxel, gemcitabine, docetaxel, gefitiniband erlotinib sensitivity between the low-risk and high-risk groups.

\section{Conclusions}

Our results reveal that glycolysis-related gene are feasible predictors of LUAD patient survival and response to therapeutics.

\section{Introduction}

Lung cancer accounts for a large proportion of cancer-related human deaths worldwide ${ }^{1,2}$. As lung adenocarcinoma (LUAD) is a common pathological type of lung cancer ${ }^{3}$, individualized therapy for LUAD 
has received increasing attention from clinicians. Because the occurrence and development of tumours are based on genetic changes ${ }^{4-6}$, the response to therapies and the overall survival times are not necessarily the same in patients of the same sex, performance status score, age and TNM stage when the influence of social, family and economic factors is removed. Therefore, exploring effective microscopic molecular biomarkers to predict the treatment response and prognosis of patients with LUAD is urgently required.

Understanding the differences in metabolism and proliferation between tumour cells and normal cells is essential for predicting the prognosis and clinical treatment response of cancer patients. Cells mainly obtain energy to fulfil their biological activities through glycometabolism, and LUAD cells are not an exception to this rule. Previous studies have revealed that the most significant metabolic change in cancer cells is the occurrence of the Warburg effect, which is manifested by increased aerobic glycolysis of tumour cells and dependence on glycolytic pathways to produce adenosine triphosphate (ATP) ${ }^{7-10}$. In view of this unique metabolic alteration in tumour cells, many targeted treatments have been tried and improved over time ${ }^{11-15}$. Moreover, an increasing number of researchers have used glycolysis-related genes to establish tumour prognosis evaluation models ${ }^{16-20}$. TMB which is defined as the number of somatic mutations per megabase $(\mathrm{Mb})$ of the interrogated genomic sequence of a tumor, and it could be used as a predictor of the efficacy of Immune checkpoint inhibitors (ICls) in multiple tumors ${ }^{21-23}$. Extensive study of TMB as prognostic molecular markers ${ }^{24-26}$ has been carried out in recent years. Since glycolysis-related genes can be used to establish an effective prognosis prediction model for LUAD, integrating TMB into the prediction model is a new and promising research approach with inherent advantages.

Here, we conducted in-depth research on gene expression data from LUAD patients in the TCGA database and used the difference of TMB to screen glycolysis-related genes. Subsequently, we found that the use of statistical tools to obtain a prognostic model and nomogram composed of glycolysis-related gene has good clinical practicality and produces a method for reliable prognosis prediction. Furthermore, the risk score was associated with the tumour immune microenvironment and could be used to estimate the sensitivity of the LUAD patients included in this study to therapeutics.

\section{Materials And Methods}

\section{Clinical information and gene expression data from patients}

The clinical characteristics, genetic mutations, and mRNA expression data from LUAD patients in the TCGA database (https://cancergenome.nih.gov/) were obtained for subsequent analyses by using Perl (version 5.32.1.1). The clinical features of 522 patients with LUAD, including gender, age, T stage (tumour), $\mathrm{N}$ stage (lymph node), M stage (metastasis), tumour stage and survival status are detailed in Table 1. 
Table 1

LUAD patient characteristics in the TCGA.

\begin{tabular}{|lll|}
\hline Clinical feature & N & $\%$ \\
\hline Gender & & \\
\hline Male & 242 & 46.4 \\
\hline Female & 280 & 53.6 \\
\hline Age (years) & & \\
\hline$\leq 65$ & 241 & 46.2 \\
\hline$>65$ & 262 & 50.2 \\
\hline Unknown & 19 & 3.6 \\
\hline Stage & & \\
\hline Stage I & 279 & 53.4 \\
\hline Stage II & 124 & 23.8 \\
\hline Stage III & 85 & 16.3 \\
\hline Stage IV & 26 & 5.0 \\
\hline Unknown & 8 & 1.5 \\
\hline T (tumor) & & \\
\hline T1 & 172 & 33.0 \\
\hline T2 & 281 & 53.8 \\
\hline T3 & 47 & 9.0 \\
\hline T4 & 19 & 3.6 \\
\hline TX & 3 & 0.6 \\
\hline N (lymph node) & & \\
\hline N0 & 335 & 64.2 \\
\hline N1 & 98 & 18.8 \\
\hline N2 & 75 & 14.4 \\
\hline N3 & 2.0 \\
\hline NX & & 0.2 \\
\hline Unknown & & \\
\hline
\end{tabular}




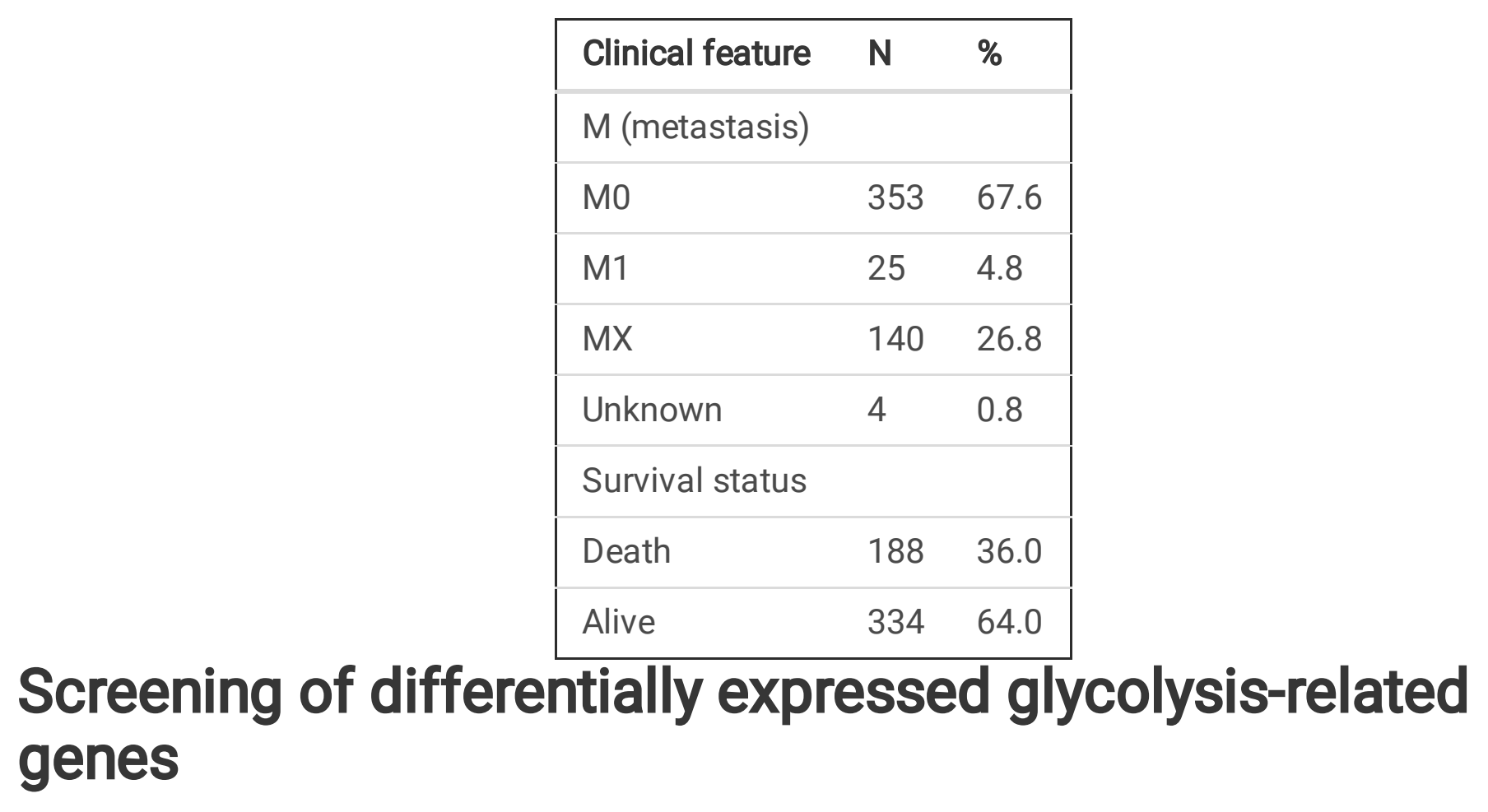

By searching glycolysis gene sets in the Gene Set Enrichment Analysis (GSEA) database (http://www.broadinstitute.org/gsea/index.jsp), a total of 326 genes were obtained. Use the gene mutation data obtained to calculate the TMB value of each sample, and divide it into high and low groups based on the median value. Subsequently, the analysis of differentially expressed glycolysisrelated genes between the high TMB group and low TMB group was executed in $\mathrm{R}$, with the following cutoff criteria: |log2fold change ( $\log F C) \mid>0.2 ;$ P-value $<0.05$; FDR (false discovery rate) $<0.05$.

\section{Gene prognosis model development}

In $\mathrm{R}$, the "survival" package was used to perform the univariate Cox regression analysis of differentially expressed genes, and the filter condition was $P<0.05$. Then, the subsequent multivariate Cox proportional hazards regression were performed. We used the following risk formula: riskscore $=\beta_{\text {gene } 1} \times$ Expression $_{\text {gene1 }}+\beta_{\text {IncRNA2 }} \times$ Expression $_{\text {gene2 }}+\beta_{\text {IncRNA3 }} \times$ Expression $_{\text {gene3 }}+\ldots+\beta_{\text {genen }} \times$ Expression $_{\text {genen }}$,

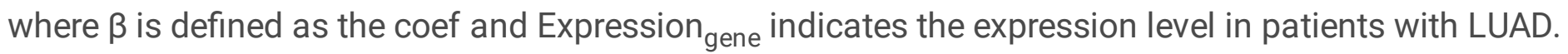
The prognostic value of genes in the model was verified by using UALCAN database (http://ualcan.path.uab.edu/index.html) and Kaplan-Meier (KM) plotter database (http://kmplot.com/analysis/). Finally, integrating GSE68465 and GSE11969, a total of 536 patient data were obtained, and they were divided into high and low risk groups using the risk score formula.

\section{Model evaluation and nomogram construction}

The ROC curve was employed to evaluate the validity of the prognostic model by using the "survival ROC" package. The LUAD patients were reclassified into a low-risk group and a high-risk group by using the cutoff value at which the best time-dependent AUC was obtained using the risk score. Then, the difference in survival times between the low-risk and high-risk groups were assessed by using the "survival" package and the "survminer" package, and the Kaplan-Meier curve was plotted to display the results directly in R. 
The Wilcoxon signed-rank test was used to obtain the risk score difference among different clinicopathological features. Then, the gene prognostic model was compared with gender, age, T stage (tumour), N stage (lymph node), M stage (metastasis), and tumour stage for independent prognostic analysis. The subsequent operations were performed with the "ggpubr" R packages. Additionally, we constructed a nomogram to improve the practicality of the model and validated it through the "rms" package.

\section{Immune microenvironment and therapeutic response}

To clarify the relationship between the immune microenvironment and the risk score, XCELL, TIMER, QUANTISEQ, MCPcounter, EPIC, CIBERSORT and CIBERSORT-ABS were used to obtain differences in the distribution of immune cells in LUAD patients from the TCGA database. The "ggplot2" R package was used to perform the procedure, the Wilcoxon signed rank test and Spearman's correlation analysis were used for the statistical analysis, and $P<0.0001$ was set as the threshold value for significance. The expression levels of immune checkpoint inhibitor-related genes were compared between the low-risk group and the high-risk group. We showed the results and the $P$ value was labelled as follows: $<0.001=$ $\star \star \star,<0.01=* \star *$, and $<0.05=*$. Subsequently, we added the pRRophetic package to evaluate clinical treatment response differences among LUAD patients grouped by the gene prognostic model by calculating the $\mathrm{IC}_{50}$ (half maximal inhibitory concentration) of commonly used drugs in $\mathrm{R}$.

\section{Results}

\section{Data processed by GSEA}

Using "glycolysis" as the search keyword, the BioCarta, Hallmark, KEGG, REACTOME and WP gene sets were selected from the Molecular Signatures Database (MSigDB) to obtain a glycolysis-related gene signature. After using the above detailed data to perform GSEA, significant differences were detected between the tumour tissue sample group and the normal tissue sample group among the gene sets (Figure 1A-D), with normalized $P$ values $<0.05$.

\section{Establishment of a glycolysis-related gene model}

By comparing the high TMB group with low TMB group, 95 differentially expressed genes were obtained (Figure 2 and Supplementary file 1). Univariate Cox analysis was performed, and we obtained 29 prognosis-related genes (Table 2), and multivariate Cox proportional hazards regression analysis was used to calculate the risk score to construct the prognostic model (Table 3 ). The prognostic was calculated as follows: $(0.00854$ * expression level of FKBP4 $)+(0.0295$ * expression level of HMMR $)+$ $(0.00651$ * expression level of B4GALT1 $)+(0.00732$ * expression level of ER01L) $+(0.000671$ * expression level of ENO1) . 
Table 2

Univariate Cox regression analysis.

\begin{tabular}{|lllll|}
\hline ID & HR & $95 \%$ CI & P-value \\
\cline { 3 - 4 } & & Lower & Upper & \\
\hline SLC16A3 & 1.021 & 1.014 & 1.028 & $2.80 \mathrm{e}-08$ \\
\hline KIF20A & 1.053 & 1.026 & 1.081 & $1.30 \mathrm{e}-04$ \\
\hline TPI1 & 1.002 & 1.001 & 1.004 & $1.48 \mathrm{e}-04$ \\
\hline DSC2 & 1.028 & 1.004 & 1.053 & $2.06 \mathrm{e}-02$ \\
\hline RPE & 1.042 & 1.015 & 1.069 & $1.88 \mathrm{e}-03$ \\
\hline PFKP & 1.007 & 1.003 & 1.011 & $3.41 \mathrm{e}-04$ \\
\hline ALDOA & 1.002 & 1.001 & 1.002 & $1.43 \mathrm{e}-03$ \\
\hline FKBP4 & 1.011 & 1.006 & 1.015 & $1.29 \mathrm{e}-05$ \\
\hline GAPDH & 1.001 & 1.000 & 1.001 & $4.30 \mathrm{e}-08$ \\
\hline HMMR & 1.062 & 1.031 & 1.092 & $4.55 \mathrm{e}-05$ \\
\hline P4HA1 & 1.007 & 1.002 & 1.013 & $8.88 \mathrm{e}-03$ \\
\hline PLOD2 & 1.010 & 1.004 & 1.015 & $3.52 \mathrm{e}-04$ \\
\hline B4GALT1 & 1.009 & 1.006 & 1.013 & $5.39 \mathrm{e}-07$ \\
\hline PYGL & 1.020 & 1.008 & 1.032 & $8.68 \mathrm{e}-04$ \\
\hline SLC2A1 & 1.006 & 1.004 & 1.009 & $9.44 \mathrm{e}-08$ \\
\hline GPI & 1.009 & 1.004 & 1.014 & $8.90 \mathrm{e}-04$ \\
\hline PGAM1 & 1.039 & 1.020 & 1.059 & $7.24 \mathrm{e}-05$ \\
\hline PGM2 & 1.053 & 1.029 & 1.078 & $1.12 \mathrm{e}-05$ \\
\hline STC1 & 1.009 & 1.004 & 1.013 & $7.94 \mathrm{e}-05$ \\
\hline DEPDC1 & 1.075 & 1.021 & 1.132 & $5.72 \mathrm{e}-03$ \\
\hline ERO1A & 1.013 & 1.008 & 1.017 & $2.61 \mathrm{e}-08$ \\
\hline TIF & 1.006 & 1.001 & 1.010 & $8.97 \mathrm{e}-03$ \\
\hline
\end{tabular}




\begin{tabular}{|lcccc|}
\hline ID & HR & $95 \%$ Cl & \multirow{2}{*}{ P-value } \\
\cline { 3 - 4 } & & Lower & Upper & \\
\hline ENO1 & 1.001 & 1.001 & 1.002 & $2.29 \mathrm{e}-04$ \\
\hline NUP37 & 1.060 & 1.017 & 1.105 & $6.28 \mathrm{e}-03$ \\
\hline PSMC4 & 1.007 & 1.001 & 1.013 & $3.36 \mathrm{e}-02$ \\
\hline
\end{tabular}

Table 3

Multivariate Cox proportional hazards regression analysis to construct a risk model.

\begin{tabular}{|lllllll|}
\hline ID & Coef & HR & \multicolumn{2}{c}{$\mathbf{9 5 \%}$ Cl } & P-value \\
\cline { 5 - 6 } & & & & Lower & Upper & \\
\hline FKBP4 & 0.00854 & 1.009 & 1.002 & 1.015 & $6.33 \mathrm{e}-03$ \\
\hline HMMR & 0.0295 & 1.030 & 0.993 & 1.068 & $1.15 \mathrm{e}-01$ \\
\hline B4GALT1 & 0.00651 & 1.007 & 1.002 & 1.011 & $1.86 \mathrm{e}-03$ \\
\hline ER01L & 0.00732 & 1.007 & 1.002 & 1.012 & $4.80 \mathrm{e}-03$ \\
\hline EN01 & 0.000671 & 1.001 & 1.000 & 1.001 & $6.71 \mathrm{e}-02$ \\
\hline
\end{tabular}

\section{Assessment of model performance}

Validation in the UALCAN database suggests that each gene in the model has prognostic value (Figure 3A-E), and similar results are also obtained in the Kaplan-Meier (KM) plotter database (Figure 3F-K). The areas under the ROCs (AUCs) of the 1-, 2-, and 3-year overall survival rate analysis for the 5-gene prognostic model were $0.673,0.671$ and 0.687 , respectively (Figure $4 A$ ). Similar verification results obtained in the GEO databas, the ROCs (AUCs) of the 1-, 2-, and 3-year overall survival rate analysis for prognostic model were $0.710,0.701$ and 0.650 , respectively (Figure 4B). Then, we used risk score cut off point to classify LUAD patients in the TCGA database into a low-risk group and a high-risk group, and the survival time was significantly different between the two groups $(p=1.085 \mathrm{e}-4)$, and the low-risk group in the GEO database also had a better prognosis $(p=2.78 \mathrm{e}-2)$ (Figure $4 \mathrm{C}$ and $\mathrm{D})$. The risk score and survival status of all patients are plotted in Figure $6 \mathrm{~A}$ and $\mathrm{B}$. Next, we conducted an independent prognostic analysis of age $(p=0.312, \mathrm{HR}=1.008,95 \% \mathrm{Cl}[0.993-1.023])$, gender $(p=0.518, \mathrm{HR}=1.102,95 \% \mathrm{Cl}$ [0.821-1.480]), stage $(p<0.001, \mathrm{HR}=1.632,95 \% \mathrm{Cl}[1.420-1.876])$ and the risk score $(p<0.001, \mathrm{HR}=$ $1.348,95 \% \mathrm{Cl}[1.263-1.438]$ ) by univariate Cox regression analysis (Figure $5 \mathrm{~A}$ ) and confirmed that stage $(p<0.001, \mathrm{HR}=1.548,95 \% \mathrm{Cl}[1.338-1.791])$ and the risk score $(p<0.001, \mathrm{HR}=1.289,95 \% \mathrm{Cl}[1.202-$ 1.383]) can be used as predictors of prognosis in LUAD patients by multivariate Cox regression analysis (Figure 5B). We also found that the AUC of the risk score of all patients was similar to the AUC of the tumour stage (Figure 5C). 


\section{Clinical evaluation and nomogram construction}

We found that $\mathrm{T}$ stage, $\mathrm{N}$ stage, and clinical stage (Figure $6 \mathrm{~A}-\mathrm{C}$ ) were significantly correlated with the risk score, while there were no differences in risk scores between gender group, age group, and $\mathrm{M}$ stage, (Figure 6D - F) through the Wilcoxon signed-rank test. Subsequently, to enhance the clinical utility of the gene model, we constructed a nomogram (Figure 7A). Risk score, age, gender and clinical stage were basic elements included in the nomogram. Moreover, the prediction results of the nomogram were generally consistent with the actual survival outcomes based on the prediction calibration curves of 1-, 2-, and 3-year survival rates (Figure 7B - D). The AUCs of the 1-, 2-, and 3-year survival rate predictions for the nomogram were $0.750,0.719$, and 0.740 (Figure $7 \mathrm{E}-\mathrm{G}$ ), respectively.

Estimation of tumour immune-related cells and molecules and patient therapeutic response with the gene assessment model

We revealed that the presence of more tumour immune-related cells, such as myeloid dendritic cell, $T$ cell $\mathrm{CD} 4+$, endothelial cell, and macrophage M2, were positively correlated with low risk, while macrophage M0, macrophage M1, and NK cell were positively associated with high risk (Figure 8A). Detailed results of the Wilcoxon signed-rank test are exhibited in Supplementary file 2. Since it is not uncommon to use immune checkpoint inhibitors to treat lung adenocarcinoma in the clinic, we aimed to clarify whether IClrelated biomarkers were related to the risk model and discovered that low risk scores were positively correlated with high expression of CD28 $(p<0.001), \operatorname{CD} 274(p<0.001)$, LAG3 $(p<0.05)$ (Figure 8B - D). Next, we explored the relationship between the risk score and the response of TCGA LUAD patients to therapeutics. Our results indicated that a low risk score correlated with a higher IC50 for medications such as cisplatin $(p=3.1 \mathrm{e}-08)$, paclitaxel $(p \otimes 2.22 \mathrm{e}-16)$, docetaxel $(p \otimes 2.22 \mathrm{e}-16)$, gemcitabine $(p=$ $1.7 \mathrm{e}-15)$, erlotinib $(p=2.5 \mathrm{e}-08)$, and gefitinib $(p=5.1 \mathrm{e}-09)$ ( Figure $9 \mathrm{~A}-\mathrm{F}$ ).

\section{Discussion}

The survival rate and quality of life of LUAD patients has improved with the development of multiple aggressive treatments, such as surgery, chemotherapy, targeted therapy and radiotherapy; however, patient responses to individualized treatment remain inconsistent. Since the occurrence and development of tumours are accompanied by changes in the expression of a variety of genes, only by fully evaluating the risks associated with gene changes can the formulation of appropriate treatment plans be improved. LUAD is a pathological type of lung malignancy and can be further divided into different therapeutic subtypes and prognostic subtypes according to the status of multiple genes. For instance, LUAD patients with EGFR mutations and ALK fusion mutations have better treatment options available and higher survival rates than patients without these mutations ${ }^{27-30}$. Moreover, as immunotherapies have been developed, researchers have found that only some patients with LUAD benefit from these treatments ${ }^{31}$. Therefore, the problem of different patient responses to treatment remains to be solved. To date, many studies have revealed that scoring can be used to assess multiple molecular markers and effectively predict patient prognosis and evaluate the potential patient response to drugs. The breast cancer 21-Gene Expression Assay is one of the most well-developed methods that can provide a prediction of patient 
prognosis, disease recurrence and tumour metastasis and can be used to guide treatment plans and assist in the development of individualized patient treatment strategies ${ }^{32}$. There have been many studies on molecular markers in lung adenocarcinoma; however, the research directions of these studies have been different (such as the development of an immune prognostic model ${ }^{33}$, an autophagy-associated gene prognostic model ${ }^{34}$, a ferroptosis-related gene prognostic model ${ }^{35}$, and a glycolysis-related gene prognostic mode ${ }^{16}$ and it is not known whether any one approach is effective for all individuals.

Therefore, constantly improving the predictive model methods will provide a variety of options for specific patients. The nomogram is a prognostic evaluation tool that can integrate several prognostic determinants, including molecular and clinicopathological parameters, and can calculate and visualize the numerical probability of clinical events with a relatively simple output and is widely used in clinical oncology ${ }^{36}$.

To obtain a more reliable prognostic model for LUAD, we used prognostic models constructed from glycolysis-related genes as a reference. First, glycolysis-related genes in LUAD were obtained, and based on the difference of TMB, differentially expressed glycolysis-related genes were selected as the cornerstone for constructing the prognostic model. Then, after performing Cox regression analyses, we found that a prognostic model composed of 5 glycolysis-related genes had better independent prognostic prediction performance, and the nomogram combined with the clinical characteristics of this model had better performance and more practical clinical application value. Meanwhile, we have also used the data in the GEO database for good verification. Since there was a difference in survival times between patients grouped according to the model, we investigated the reason for this difference. The results of our in-depth study revealed that there were differences in tumour pathological characteristics and immune responses among patients grouped according to glycolysis-related genes, as well as differences in sensitivity to therapeutic drugs. Therefore, we have presented sufficient evidence to demonstrate that the gene model obtained by the method in this paper has a a better auxiliary effect in the prediction of LUAD patient response to clinical treatment.

More recently, numerous studies have been conducted on the use of gene or IncRNA to construct a prognostic model for LUAD. Sun et al. reported that immune-related genes could be used to construct a prognostic model. However, nomogram was not combined with the model and clinicopathological characteristics, so it was impossible to evaluate the effects of age, gender and stage for a specific clinical patient using this model ${ }^{37}$. Although $\mathrm{Xu}$ et al. obtained prognostic biomarkers by analysing the tumor microenvironment of LUAD, they did not calculate the AUC value of the model ${ }^{38}$. Most of the risk models are based on the detection of the expression level of the molecule of interest and the calculation of the total risk score to determine the prognosis of the patient. The first requirement is to judge the accuracy of the model before considering whether it can be used in clinical practice. Li et al. found that RNA binding proteins could be prognostic signatures for LUAD, the model obtained had good prediction performance, and a nomogram was also constructed ${ }^{39}$. However, the differences in the immune microenvironment between the groups based on that model have not been further explored. It is well known that the prognosis of patients is related to a variety of factors, and the aforementioned model is of limited use for 
predicting survival time. Additionally, the clinical treatment plan for patients is somewhat volatile. Therefore, a model is more valuable if it also has the ability to predict patient response to medication. Wu et al. validated a LUAD patient prognostic biomarker constructed using autophagy-related long noncoding RNAs ${ }^{40}$, but the risk model did not specify the 1-year, 2-year and 3-year survival rate AUCs in detail, nor did it analyze the relationship with clinicopathological features. Zhang et al. also constructed a prognostic model based on glycolysis-related genes, but it did not specify the AUC value of 1, 2 and 3 years, did not use TMB for differential analysis, and did not verify $i^{41}$. And the number of genes in the model is more than that in this study. Our prognostic model based on the metabolic characteristics of tumours has the following characteristics. First, the theoretical basis is sufficient. Glycolysis, as a metabolic characteristic of common tumour changes, has been confirmed to be relevant by many researchers. Second, the data screening was reasonable, and the data processed by the Cox regression analysis were more reliable. Finally, the assessment of the nomogram and its ability to predict patient drug sensitivity provides better clinical applicability.

Although the model we constructed has the above advantages, there are also some shortcomings. We are not in a position to conduct in vitro studies to further verify the function of these genes. FKBP4, HMMR and B4GALT1 are associated with the occurrence and development of LUAD or the survival prognosis of

patients ${ }^{17,41}$. ERO1L was found to be a potential biomarker in LUAD and shapes the immune-suppressive tumor microenvironment. ENO1 was confirmed to be able to promote glycolysis and tumor progression in lung adenocarcinoma through CircRNA-ENO $1^{42}$. When better experimental research resources become available in the future, the predictive ability of these genes can be verified in more LUAD patients.

\section{Conclusion}

In summary, a LUAD risk prognostic signature of 5 glycolysis-related genes was identified in this study. For predicting the survival time of patients with LUAD and their potential response to therapeutics, the model obtained in this study has excellent performance. This is the first study to predict the survival and drug response of LUAD patients by combining glycolysis-related genes with TMB. Furthermore, the combination of glycolysis-related genes and immune responses could not only enhance the accuracy of the model but also lead to a new direction in immunotherapy.

\section{Declarations}

\section{Conflict of interest}

The authors declare that the research was conducted in the absence of any commercial or financial relationships that could be construed as a potential conflict of interest.

\section{Acknowledgments}




\section{Author contributions}

Rongchang Zhao wrote the manuscript. Rongchang Zhao, Dan Ding, and Xiujuan Wang performed the statistical analysis. Yan Ding, Rongbo Han, and Chunrong Zhu prepared the figure and wrote the figure legend. All authors approved the final version to be published.

\section{References}

1. Barta JA, Powell CA, Wisnivesky JP. Global Epidemiology of Lung Cancer. Ann Glob Health. 2019;85(1).https://doi.org/10.5334/aogh.2419.

2. Bade BC, Dela Cruz CS. Lung C. 2020: Epidemiology, Etiology, and Prevention. Clin Chest Med. 2020;41(1):1-24.https://doi.org/10.1016/j.ccm.2019.10.001.

3. Travis WD, Brambilla E, Burke AP, Marx A, Nicholson AG. Introduction to The 2015 World Health Organization Classification of Tumors of the Lung, Pleura, Thymus, and Heart. J Thorac Oncol. 2015;10(9):1240-2. .https://doi.org/10.1097/jto.0000000000000663.

4. Vogelstein B, Papadopoulos N, Velculescu VE, Zhou S, Diaz LA Jr, Kinzler KW. Cancer genome landscapes. Science. 2013;339(6127):1546-58. .https://doi.org/10.1126/science.1235122.

5. Zhu K, Liu Q, Zhou Y, Tao C, Zhao Z, Sun J, et al. Oncogenes and tumor suppressor genes: comparative genomics and network perspectives. BMC Genomics. 2015;16 Suppl 7(Suppl 7):S8.https://doi.org/10.1186/1471-2164-16-s7-s8

6. Comprehensive genomic characterization of squamous cell lung cancers. Nature. 2012;489(7417):519-25. .https://doi.org/10.1038/nature11404.

7. Koppenol WH, Bounds PL, Dang CV. Otto Warburg's contributions to current concepts of cancer metabolism. Nat Rev Cancer. 2011;11(5):325-37. .https://doi.org/10.1038/nrc3038.

8. Xu XD, Shao SX, Jiang HP, Cao YW, Wang YH, Yang XC, et al. Warburg effect or reverse Warburg effect? A review of cancer metabolism. Oncol Res Treat. 2015;38(3):117-22. .https://doi.org/10.1159/000375435.

9. Schwartz L, Supuran CT, Alfarouk KO. The Warburg Effect and the Hallmarks of Cancer. Anticancer Agents Med Chem. 2017;17(2):164-70. .https://doi.org/10.2174/1871520616666161031143301.

10. Vaupel $P$, Schmidberger $H$, Mayer A. The Warburg effect: essential part of metabolic reprogramming and central contributor to cancer progression. Int J Radiat Biol. 2019;95(7):912-9. .https://doi.org/10.1080/09553002.2019.1589653.

11. Danial NN, Gramm CF, Scorrano L, Zhang CY, Krauss S, Ranger AM, et al. BAD and glucokinase reside in a mitochondrial complex that integrates glycolysis and apoptosis. Nature. 2003;424(6951):952-6. .https://doi.org/10.1038/nature01825. 
12. Xu RH, Pelicano H, Zhou Y, Carew JS, Feng L, Bhalla KN, et al. Inhibition of glycolysis in cancer cells: a novel strategy to overcome drug resistance associated with mitochondrial respiratory defect and hypoxia. Cancer Res. 2005;65(2):613-21.

13. Coy JF, Dressler D, Wilde J, Schubert P. Mutations in the transketolase-like gene TKTL1: clinical implications for neurodegenerative diseases, diabetes and cancer. Clin Lab. 2005;51(5-6):257-73.

14. Pelicano H, Martin DS, Xu RH, Huang P. Glycolysis inhibition for anticancer treatment. Oncogene. 2006;25(34):4633-46. https://doi.org/10.1038/sj.onc.1209597.

15. Yang J, Ren B, Yang G, Wang $H$, Chen G, You L, et al. The enhancement of glycolysis regulates pancreatic cancer metastasis. Cell Mol Life Sci. 2020;77(2):305-21.

.https://doi.org/10.1007/s00018-019-03278-z.

16. Liu J, Li S, Feng G, Meng H, Nie S, Sun R, et al. Nine glycolysis-related gene signature predicting the survival of patients with endometrial adenocarcinoma. Cancer Cell Int. 2020;20:183. https://doi.org/10.1186/s12935-020-01264-1.

17. Zhang L, Zhang Z, Yu Z. Identification of a novel glycolysis-related gene signature for predicting metastasis and survival in patients with lung adenocarcinoma. J Transl Med. 2019;17(1):423. https://doi.org/10.1186/s12967-019-02173-2.

18. Wu C, Cai X, Yan J, Deng A, Cao Y, Zhu X. Identification of Novel Glycolysis-Related Gene Signatures Associated With Prognosis of Patients With Clear Cell Renal Cell Carcinoma Based on TCGA. Front Genet. 2020;11:589663. https://doi.org/10.3389/fgene.2020.589663.

19. Tang J, Luo Y, Wu G. A glycolysis-related gene expression signature in predicting recurrence of breast cancer. Aging. 2020;12(24):24983-94. .https://doi.org/10.18632/aging.103806.

20. Zhou W, Zhang S, Cai Z, Gao F, Deng W, Wen Y, et al. A glycolysis-related gene pairs signature predicts prognosis in patients with hepatocellular carcinoma. PeerJ. 2020;8:e9944. https://doi.org/10.7717/peerj.9944.

21. Snyder A, Makarov V, Merghoub T, Yuan J, Zaretsky JM, Desrichard A, et al. Genetic basis for clinical response to CTLA-4 blockade in melanoma. N Engl J Med. 2014;371(23):2189-99. .https://doi.org/10.1056/NEJMoa1406498.

22. Rizvi NA, Hellmann MD, Snyder A, Kvistborg P, Makarov V, Havel JJ, et al. Cancer immunology. Mutational landscape determines sensitivity to PD-1 blockade in non-small cell lung cancer. Science. 2015;348(6230):124-8. .https://doi.org/10.1126/science.aaa1348.

23. Dong ZY, Zhong WZ, Zhang XC, Su J, Xie Z, Liu SY, et al. Potential Predictive Value of TP53 and KRAS Mutation Status for Response to PD-1 Blockade Immunotherapy in Lung Adenocarcinoma. Clin Cancer Res. 2017;23(12):3012-24. .https://doi.org/10.1158/1078-0432.Ccr-16-2554.

24. Lv J, Zhu Y, Ji A, Zhang Q, Liao G. Mining TCGA database for tumor mutation burden and their clinical significance in bladder cancer. Biosci Rep. 2020;40(4).https://doi.org/10.1042/bsr20194337.

25. Zhang L, Li B, Peng Y, Wu F, Li Q, Lin Z, et al. The prognostic value of TMB and the relationship between TMB and immune infiltration in head and neck squamous cell carcinoma: A gene 
expression-based study. Oral Oncol. 2020;110:104943.

https://doi.org/10.1016/j.oraloncology.2020.104943.

26. Kang K, Xie F, Mao J, Bai Y, Wang X. Significance of Tumor Mutation Burden in Immune Infiltration and Prognosis in Cutaneous Melanoma. Front Oncol. 2020;10:573141.

https://doi.org/10.3389/fonc.2020.573141.

27. Shan L, Wang Z, Guo L, Sun H, Qiu T, Ling Y, et al. Concurrence of EGFR amplification and sensitizing mutations indicate a better survival benefit from EGFR-TKI therapy in lung adenocarcinoma patients. Lung Cancer. 2015;89(3):337-42. .https://doi.org/10.1016/j.lungcan.2015.06.008.

28. Zhu JQ, Zhong WZ, Zhang GC, Li R, Zhang XC, Guo AL, et al. Better survival with EGFR exon 19 than exon 21 mutations in gefitinib-treated non-small cell lung cancer patients is due to differential inhibition of downstream signals. Cancer Lett. 2008;265(2):307-17. .https://doi.org/10.1016/j.canlet.2008.02.064.

29. Khan M, Lin J, Liao G, Tian Y, Liang Y, Li R, et al. ALK Inhibitors in the Treatment of ALK Positive NSCLC. Front Oncol. 2018;8:557. https://doi.org/10.3389/fonc.2018.00557.

30. Kang J, Zhang XC, Chen HJ, Zhong WZ, Xu Y, Su J, et al. Complex ALK Fusions Are Associated With Better Prognosis in Advanced Non-Small Cell Lung Cancer. Front Oncol. 2020;10:596937. https://doi.org/10.3389/fonc.2020.596937.

31. Herbst RS, Giaccone G, de Marinis F, Reinmuth N, Vergnenegre A, Barrios CH, et al. Atezolizumab for First-Line Treatment of PD-L1-Selected Patients with NSCLC. N Engl J Med. 2020;383(14):1328-39. .https://doi.org/10.1056/NEJMoa1917346.

32. Sparano JA, Gray RJ, Makower DF, Pritchard KI, Albain KS, Hayes DF, et al. Adjuvant Chemotherapy Guided by a 21-Gene Expression Assay in Breast Cancer. N Engl J Med. 2018;379(2):111-21. https://doi.org/10.1056/NEJMoa1804710.

33. Luo C, Lei M, Zhang Y, Zhang Q, Li L, Lian J, et al. Systematic construction and validation of an immune prognostic model for lung adenocarcinoma. J Cell Mol Med. 2020;24(2):1233-44. https://doi.org/10.1111/jcmm.14719.

34. Wang X, Yao S, Xiao Z, Gong J, Liu Z, Han B, et al. Development and validation of a survival model for lung adenocarcinoma based on autophagy-associated genes. J Transl Med. 2020;18(1):149. https://doi.org/10.1186/s12967-020-02321-z.

35. Gao X, Tang M, Tian S, Li J, Liu W. A ferroptosis-related gene signature predicts overall survival in patients with lung adenocarcinoma. Future Oncol. 2021;17(12):1533-44. .https://doi.org/10.2217/fon-2020-1113.

36. Balachandran VP, Gonen M, Smith JJ, DeMatteo RP. Nomograms in oncology: more than meets the eye. Lancet Oncol. 2015;16(4):e173-80. https://doi.org/10.1016/s1470-2045(14)71116-7.

37. Sun S, Guo W, Wang Z, Wang X, Zhang G, Zhang H, et al. Development and validation of an immunerelated prognostic signature in lung adenocarcinoma. Cancer Med. 2020;9(16):5960-75. https://doi.org/10.1002/cam4.3240. 
38. Xu ZY, Zhao M, Chen W, Li K, Qin F, Xiang WW, et al. Analysis of prognostic genes in the tumor microenvironment of lung adenocarcinoma. PeerJ. 2020;8:e9530. .https://doi.org/10.7717/peerj.9530.

39. Li W, Gao LN, Song PP, You CG. Development and validation of a RNA binding protein-associated prognostic model for lung adenocarcinoma. Aging. 2020;12(4):3558-73. .https://doi.org/10.18632/aging.102828.

40. Wu L, Wen Z, Song Y, Wang L. A novel autophagy-related IncRNA survival model for lung adenocarcinoma. J Cell Mol Med. 2021;25(12):5681-90. https://doi.org/10.1111/jcmm.16582.

41. Jia S, Li L, Xie L, Zhang W, Zhu T, Qian B. Transcriptome Based Estrogen Related Genes Biomarkers for Diagnosis and Prognosis in Non-small Cell Lung Cancer. Front Genet. 2021;12:666396. https://doi.org/10.3389/fgene.2021.666396.

42. Zhou J, Zhang S, Chen Z, He Z, Xu Y, Li Z. CircRNA-ENO1 promoted glycolysis and tumor progression in lung adenocarcinoma through upregulating its host gene ENO1. Cell Death Dis. 2019;10(12):885. https://doi.org/10.1038/s41419-019-2127-7.

\section{Figures}


A
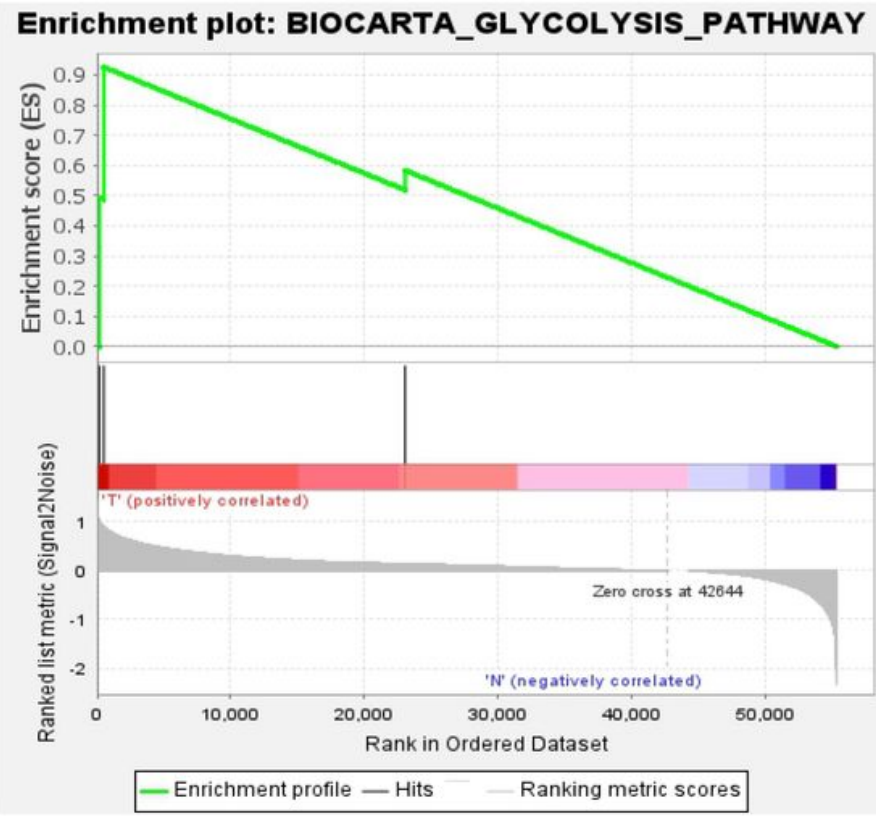

$\mathrm{C}$

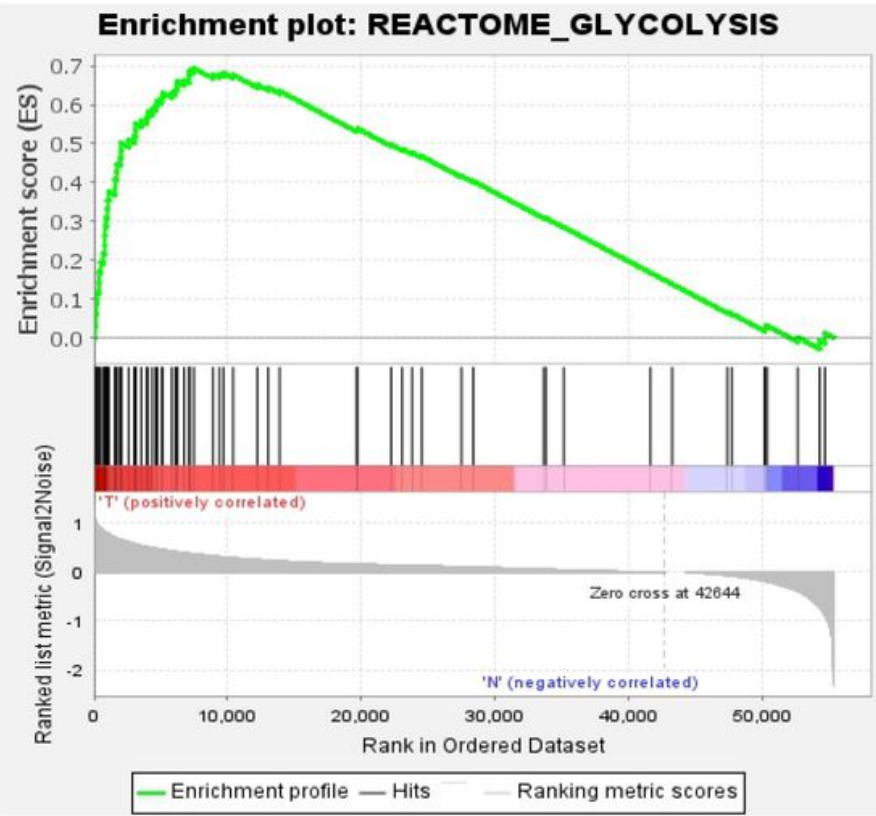

B

Enrichment plot: HALLMARK_GLYCOLYSIS

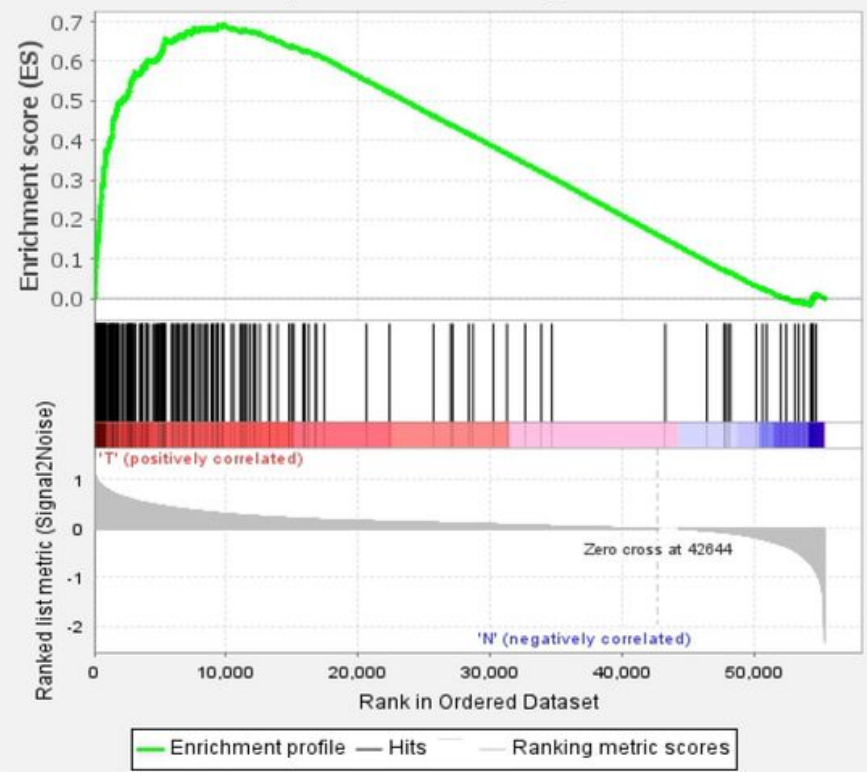

$\mathrm{D}$

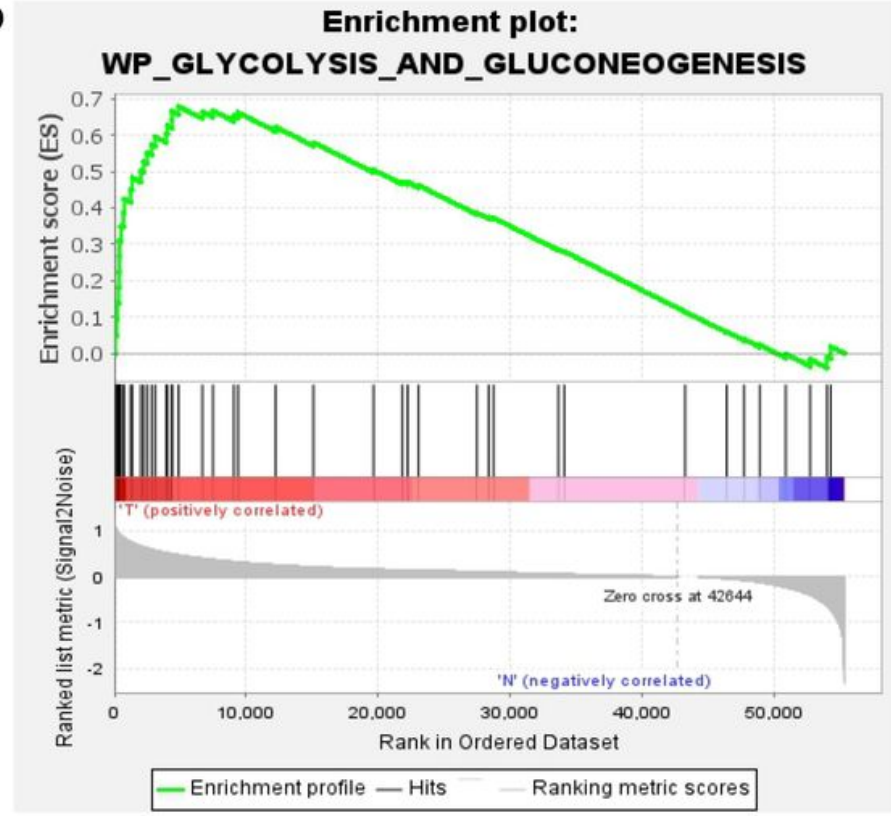

Figure 1

GSEA revealed that four gene sets were significantly enriched: (A) BioCarta glycolysis, (B) glycolysis, (C) REACTOME glycolysis and (D) WP glycolysis and gluconeogenesis. 


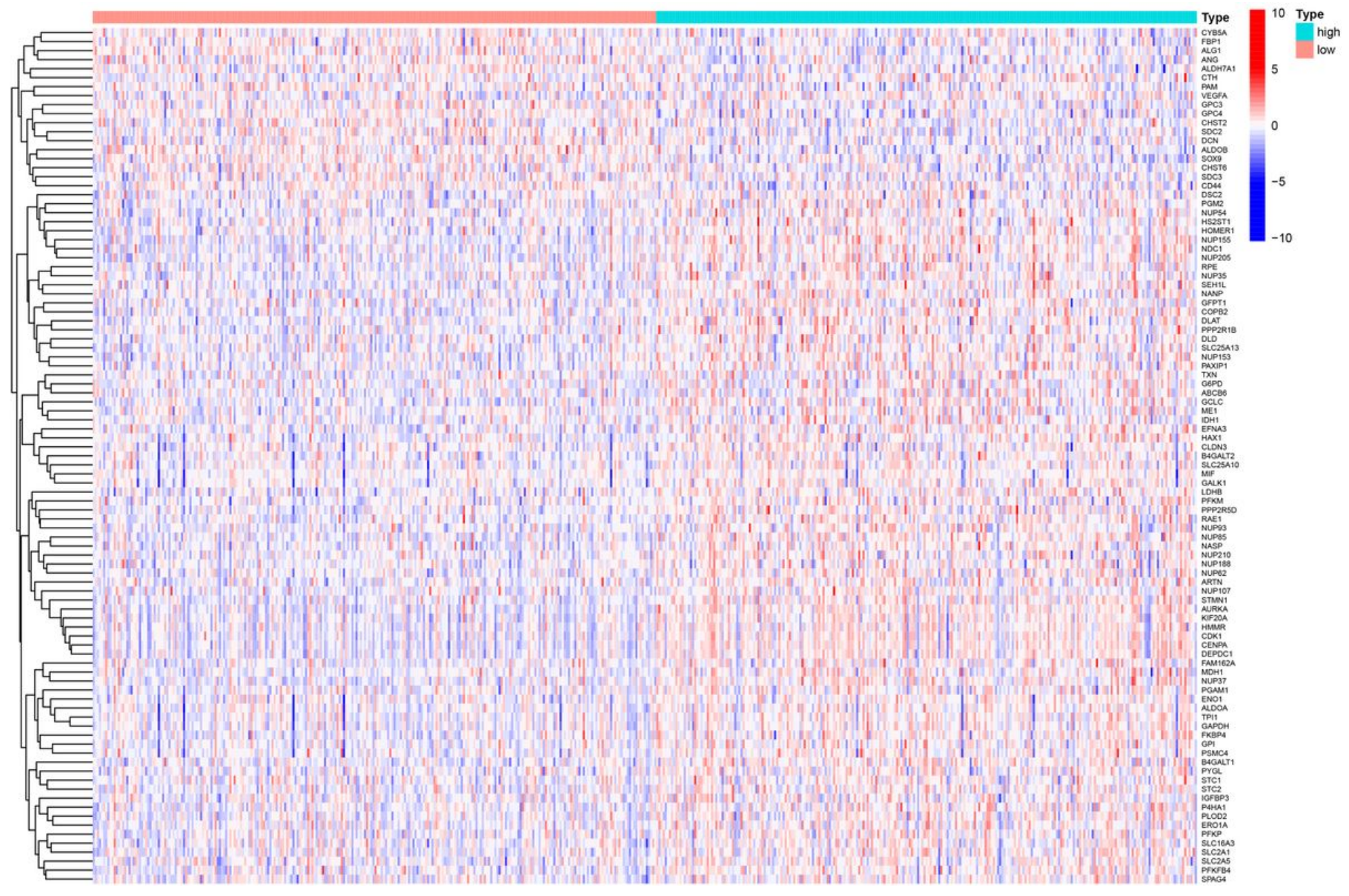

Figure 2

Heatmap diagram of differentially expressed glycolysis-related genes, by comparing the high TMB group with low TMB group 

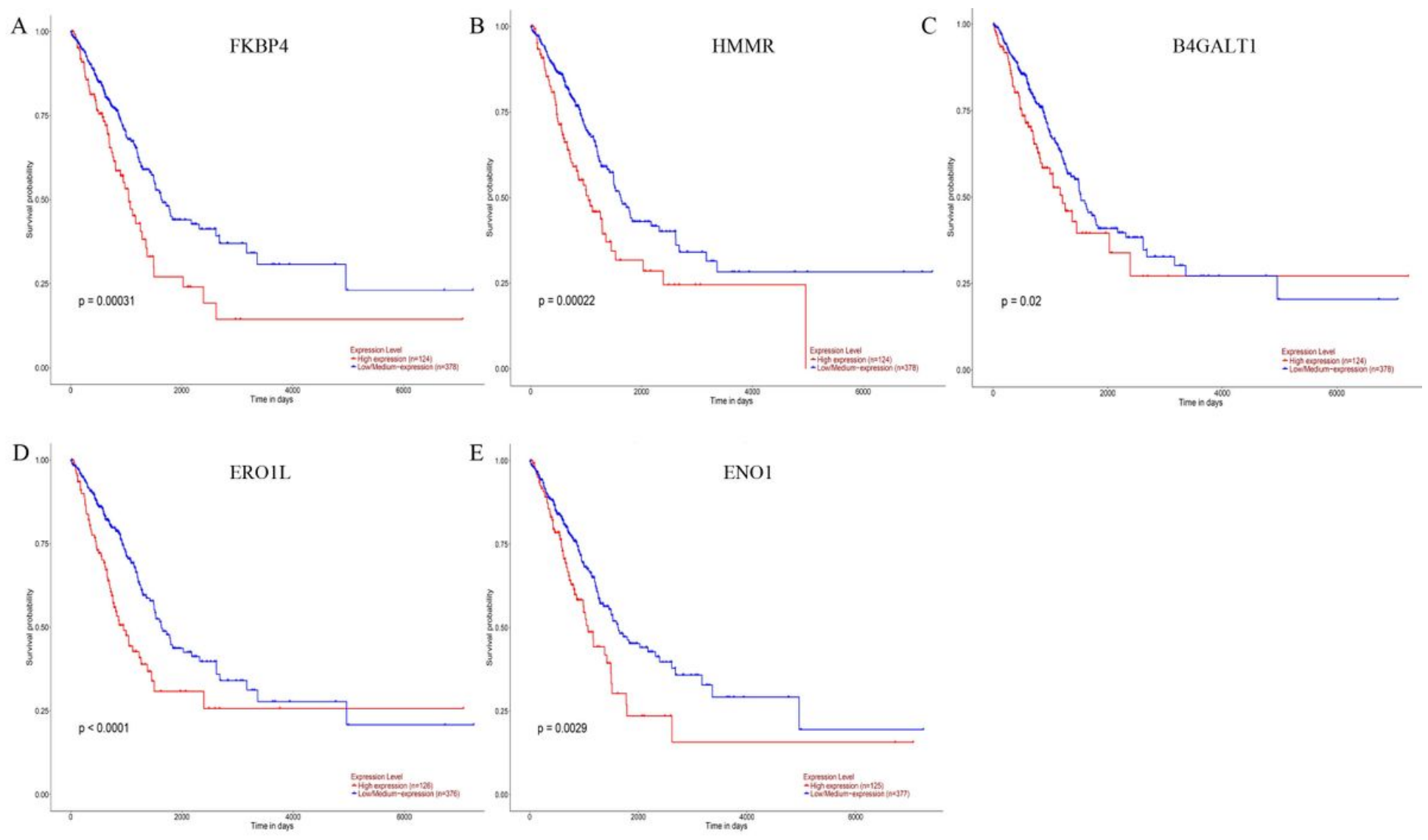

Figure 3

Prognostic validation of genes in the prognostic model. (A) FKBP4, (B) HMMR, (C)B4GALT1, (D)ER01L, and (E)ENO1 all have the ability to predict survival time, in UALCAN database. 

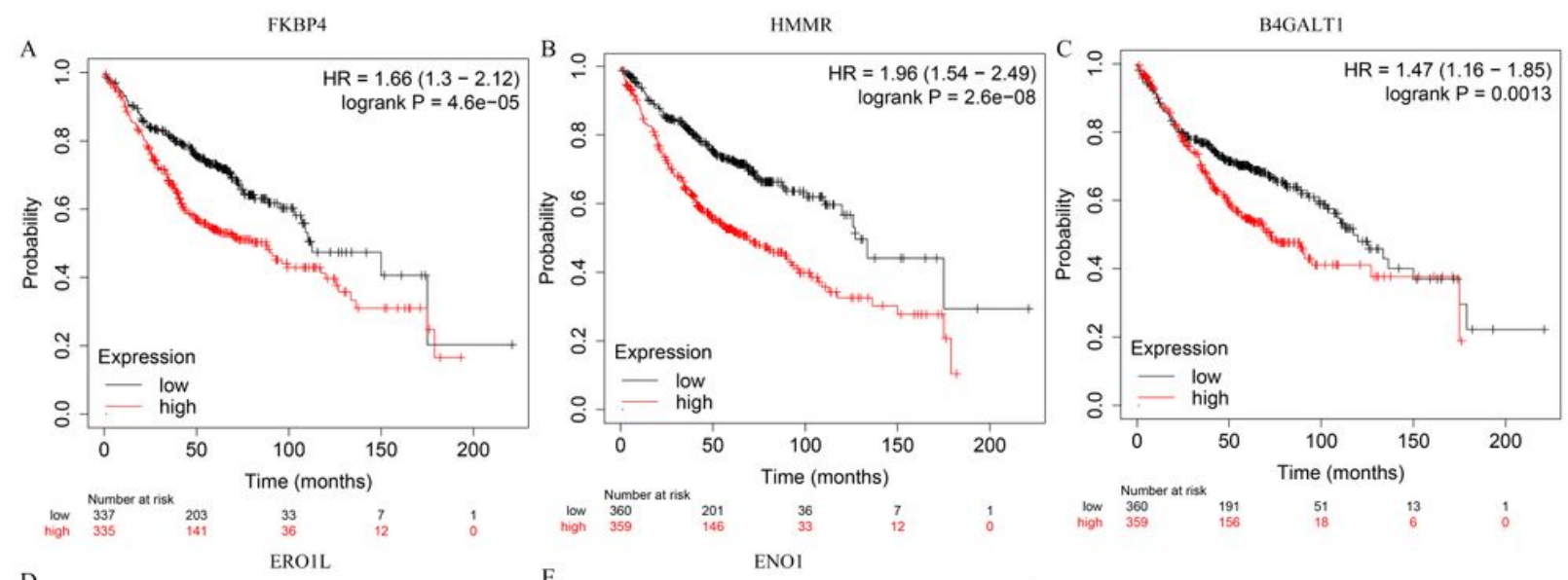

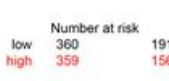
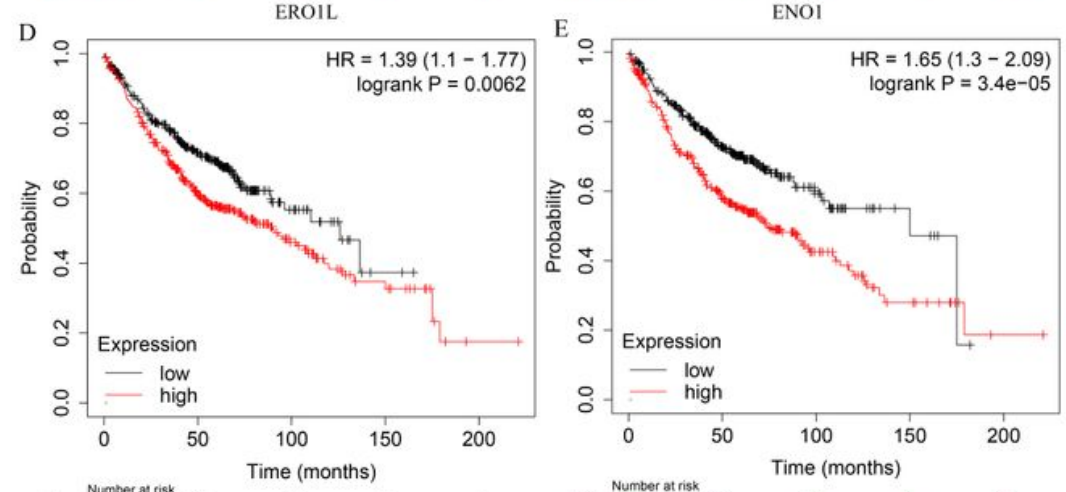

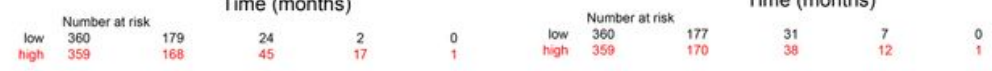

\section{Figure 4}

Prognostic validation of genes in the prognostic model. (A) FKBP4, (B) HMMR, (C)B4GALT1, (D)ERO1L, and (E)ENO1 also all have the ability to predict survival time, in Kaplan-Meier (KM) plotter database. 


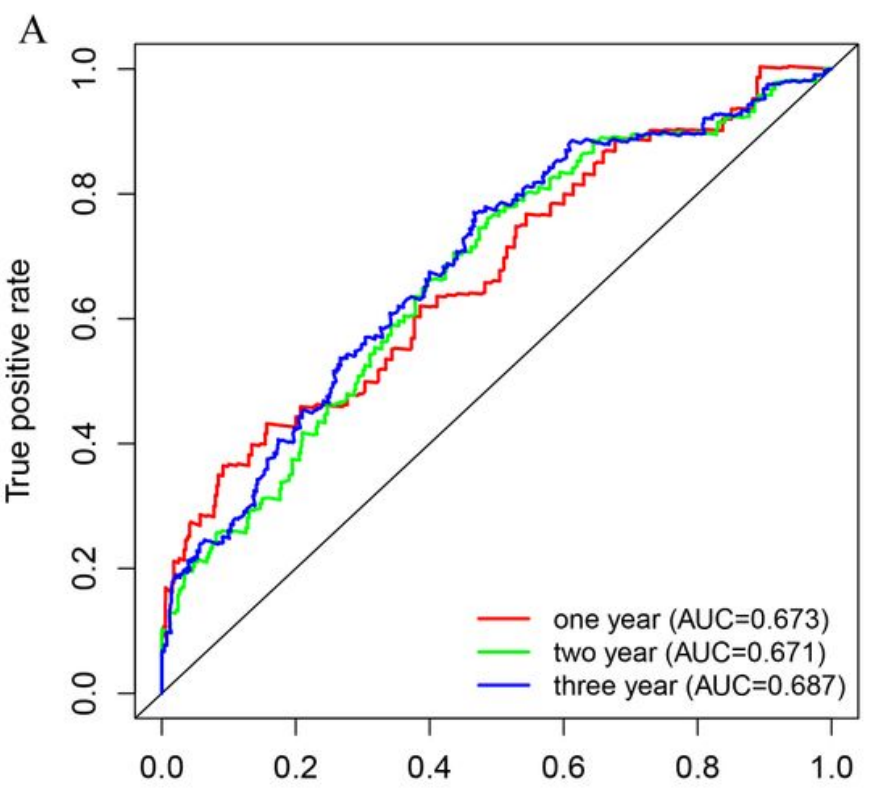

B

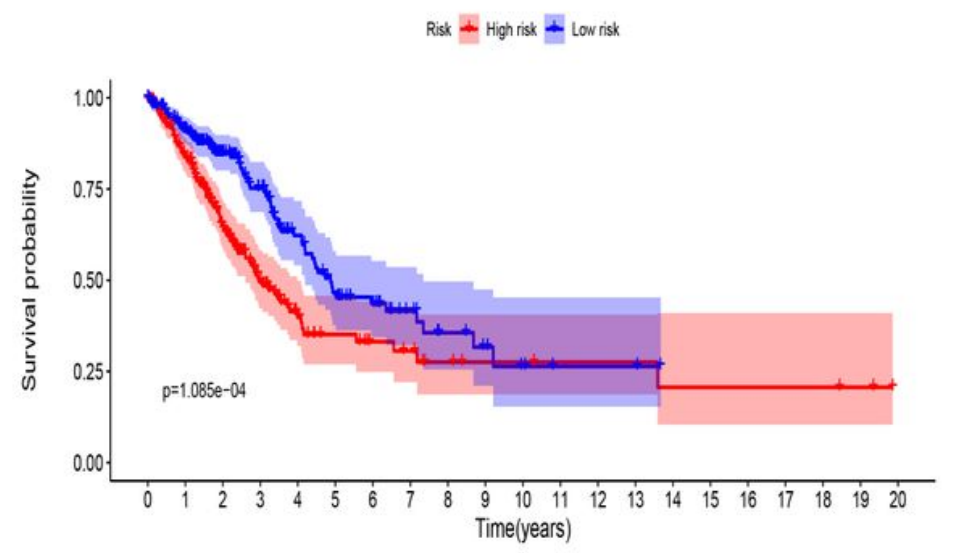

False positive rate

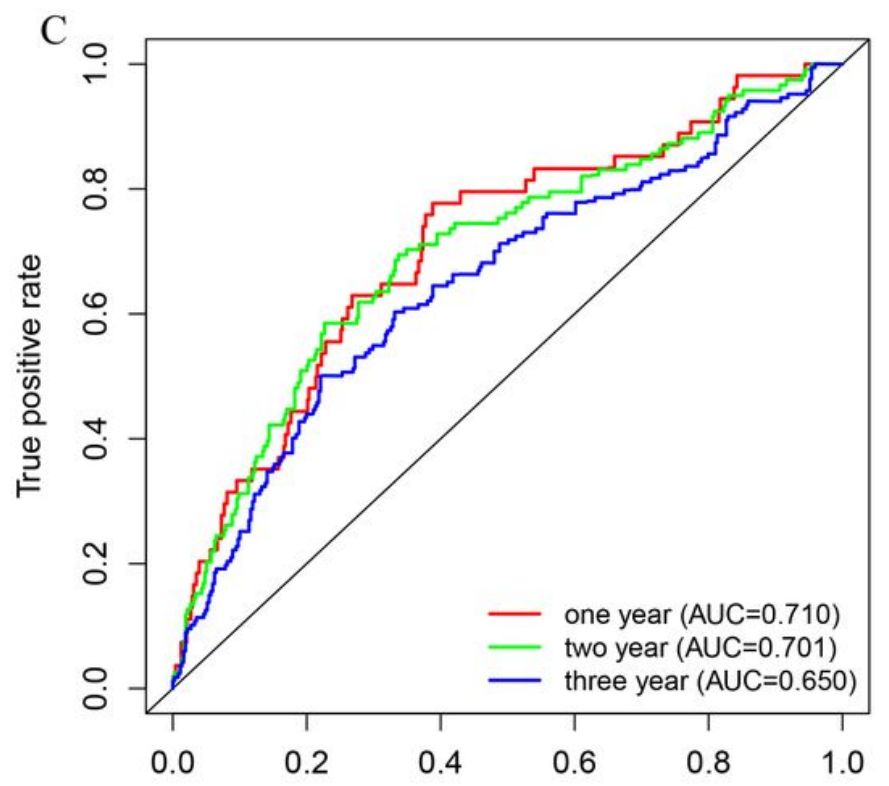

D

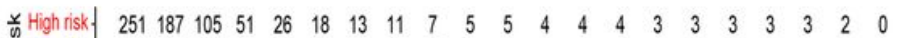

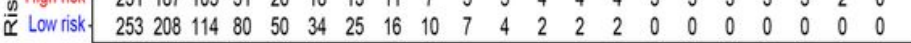
$\begin{array}{llllllllllllllllllll}1 & 1 & 3 & 4 & 5 & 6 & 7 & 8 & 9 & 10 & 11 & 12 & 13 & 14 & 15 & 16 & 17 & 18 & 19 & 20 \\ \text { Time(years) }\end{array}$

D

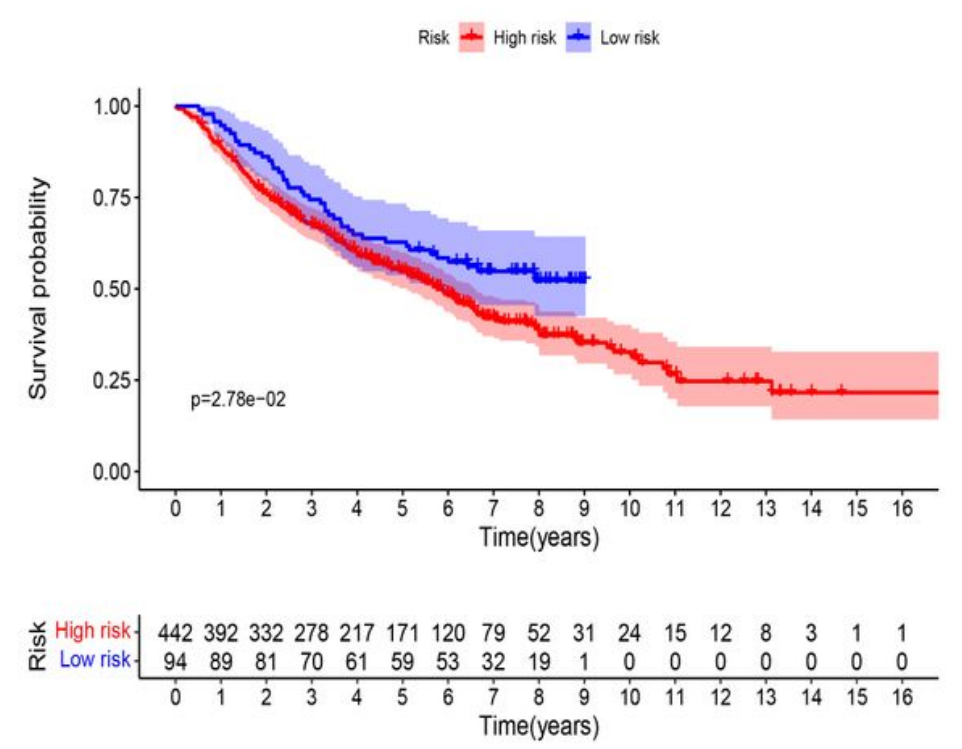

False positive rate

Figure 5

Evaluation and validation of the glycolysis-related gene risk model. (A) AUC values of 1-, 2-, and 3-year survival rates are shown in the ROC curve, by integrating TCGA database data. (B) TAUC values of 1-, 2-, and 3-year survival rates are shown in the ROC curve, by integrating GEO database data. (C) Kaplan-Meier survival curves of patients based on the risk score of 5 glycolysis-related genes, in TCGA database. (D) Kaplan-Meier survival curves of patients based on the risk score of 5 glycolysis-related genes, in GEO database. 


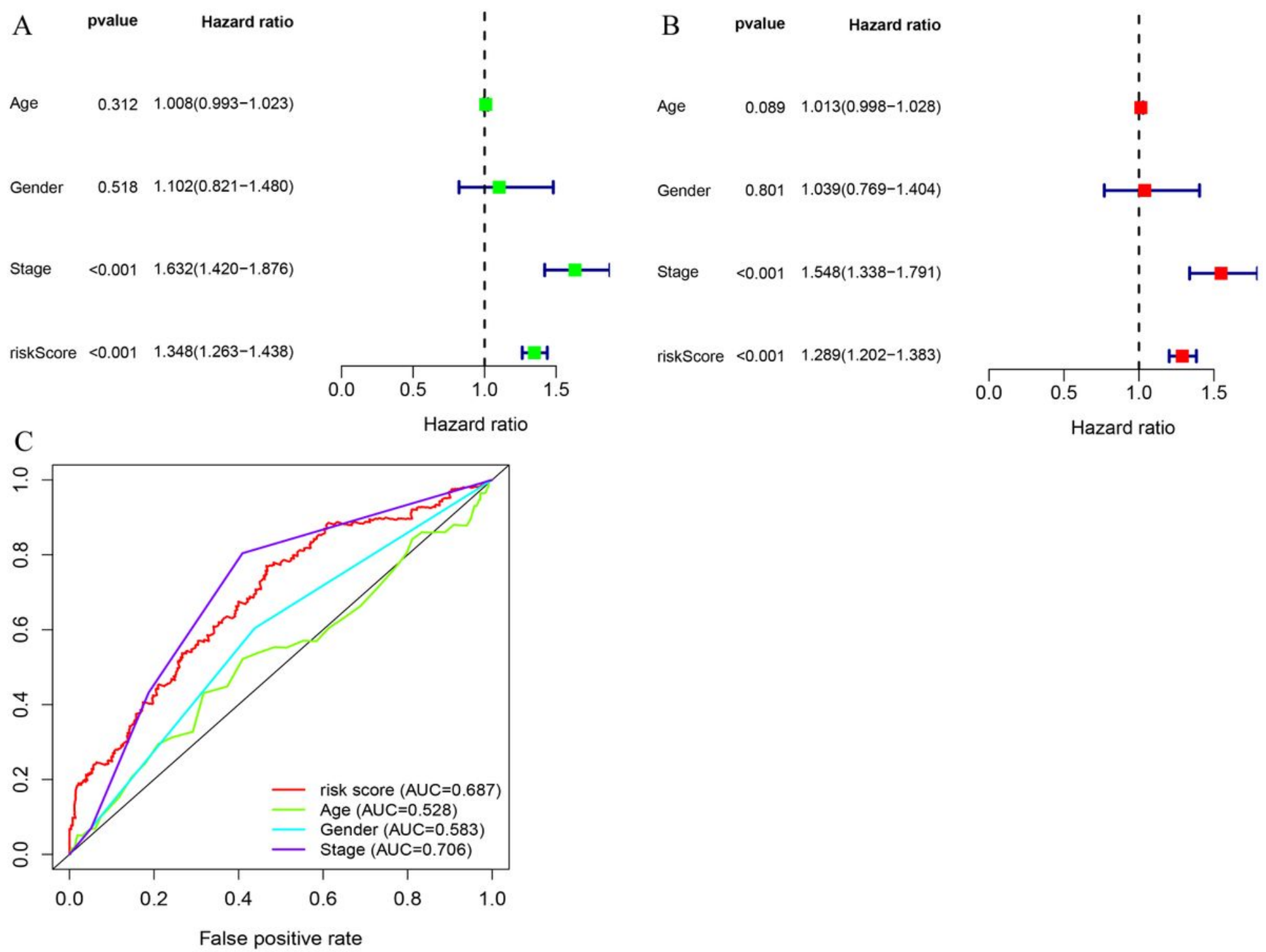

\section{Figure 6}

Comparison with clinicopathologic features. (A) Univariate cox regression analyses. (B) Multivariate cox regression analyses. (C) AUC of the risk score, age, gender and stage. 

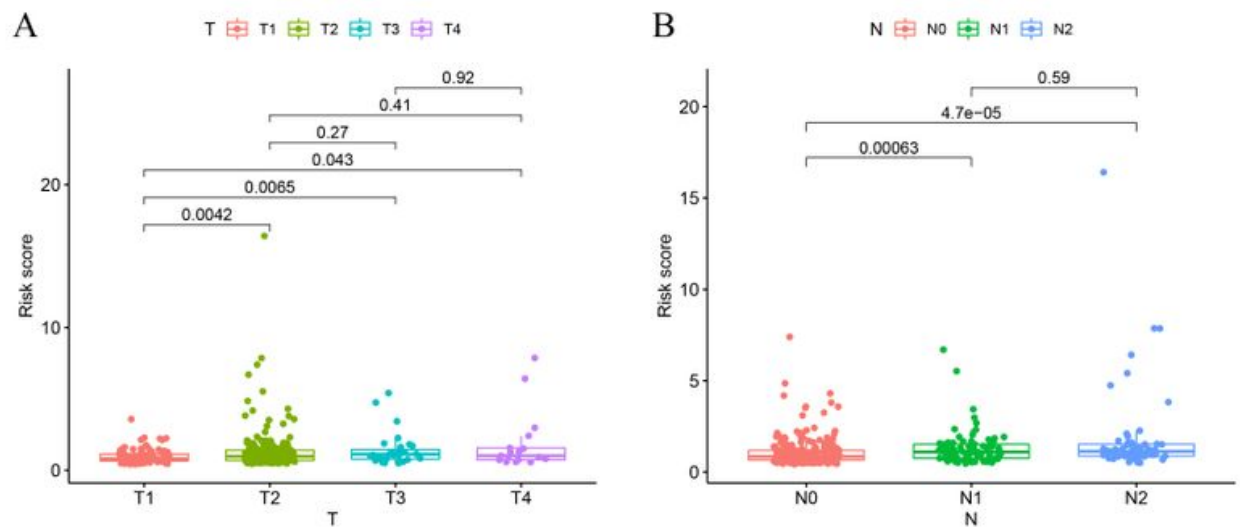

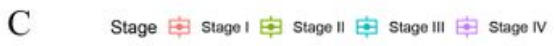
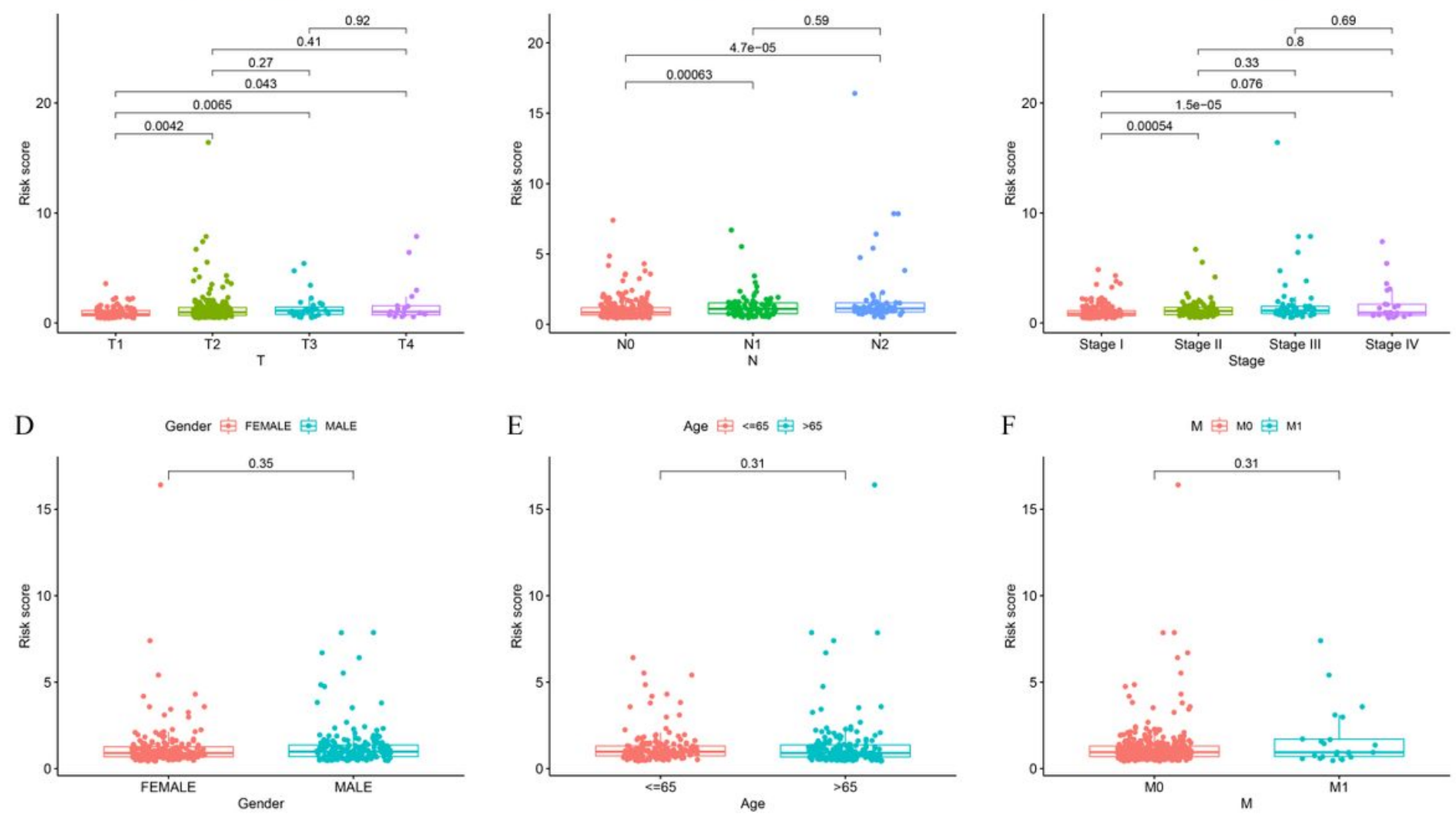

\section{Figure 7}

Use risk assessment models for clinical evaluation. (A) A strip chart for information overview. The scatter diagram of (A) T stage, (B) N stage, (C) tumour stage, (D) gender, (E) age, and (F) M stage. 

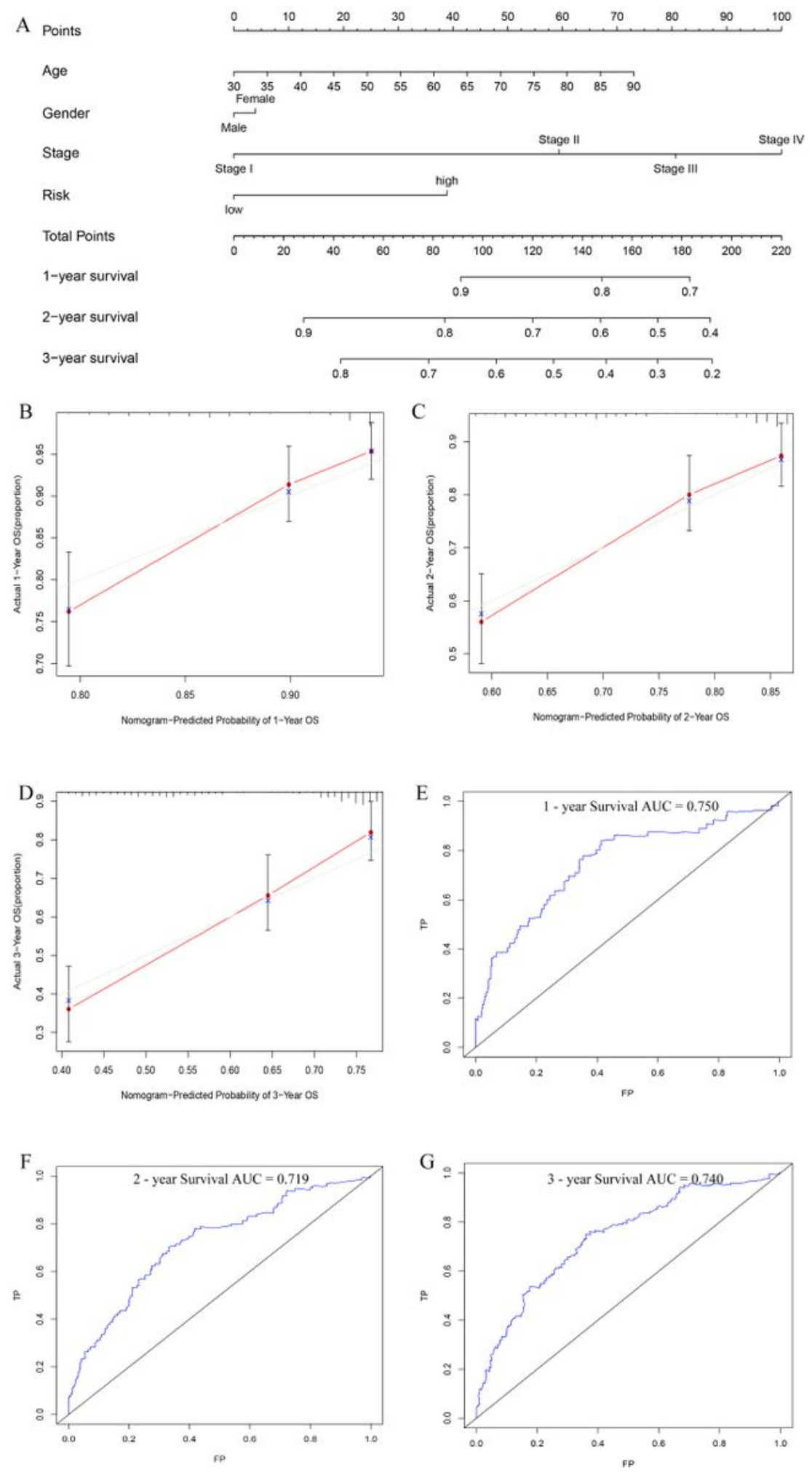

\section{Figure 8}

A nomogram for survival prediction was constructed and evaluated. (A) The nomogram for predicting the 1-, 2-, and 3-year survival rate of LUAD patients. The consistency of the actual proposal and nomogrampredicted probability of OS at (B) 1, (C) 2 and (D) 3 years. AUC calculated for (E) 1, (F) 2 and (G) 3 years based on the nomogram. 

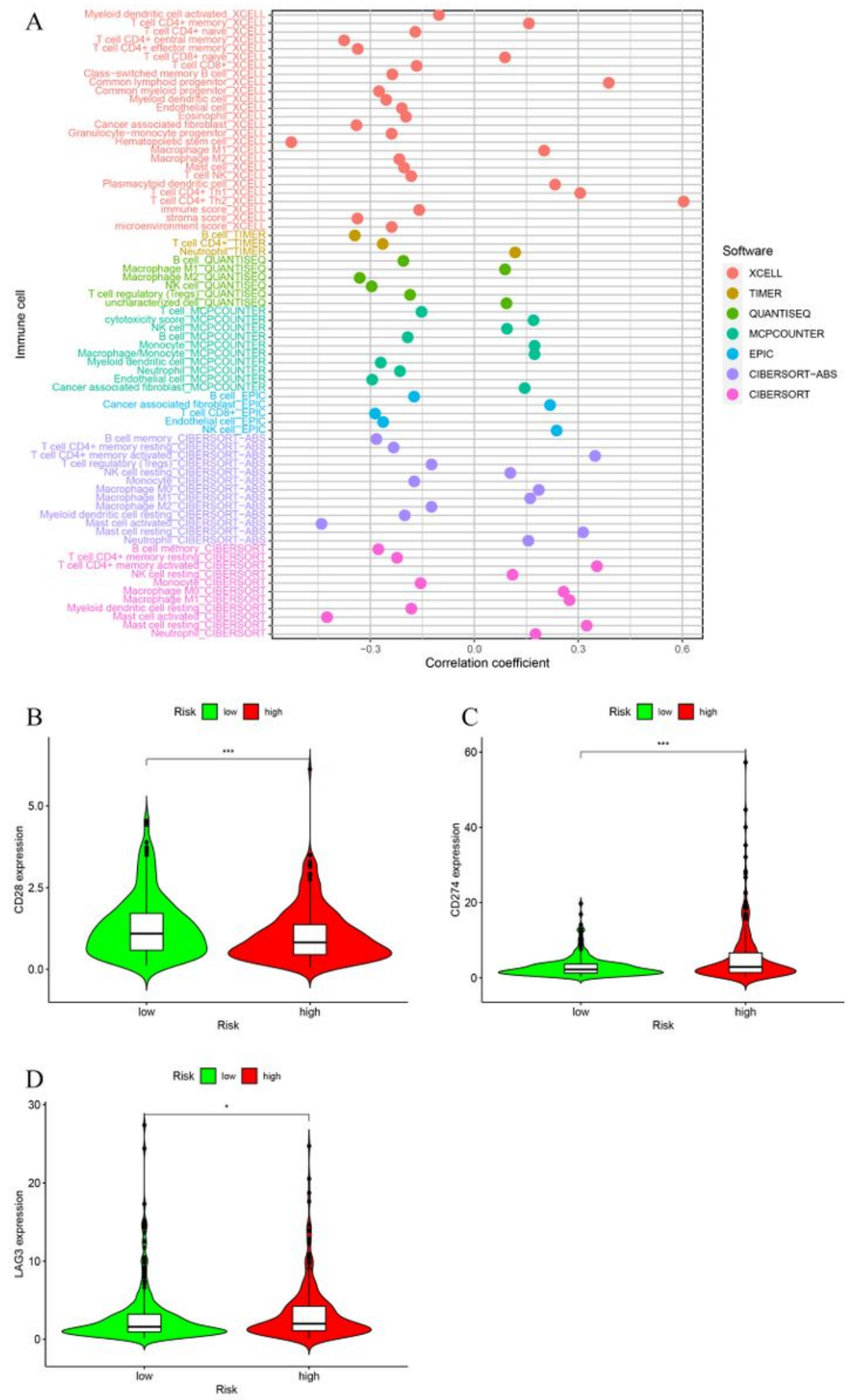

\section{Figure 9}

The risk model was used to evaluate tumour-infiltrating cells and immune costimulatory molecules. (A) Correlation between immune cells and the risk score was obtained using different methods. A low risk score was positively related to the upregulation of (B) CD28, (C) CD274, and (D) LAG3 expression. 

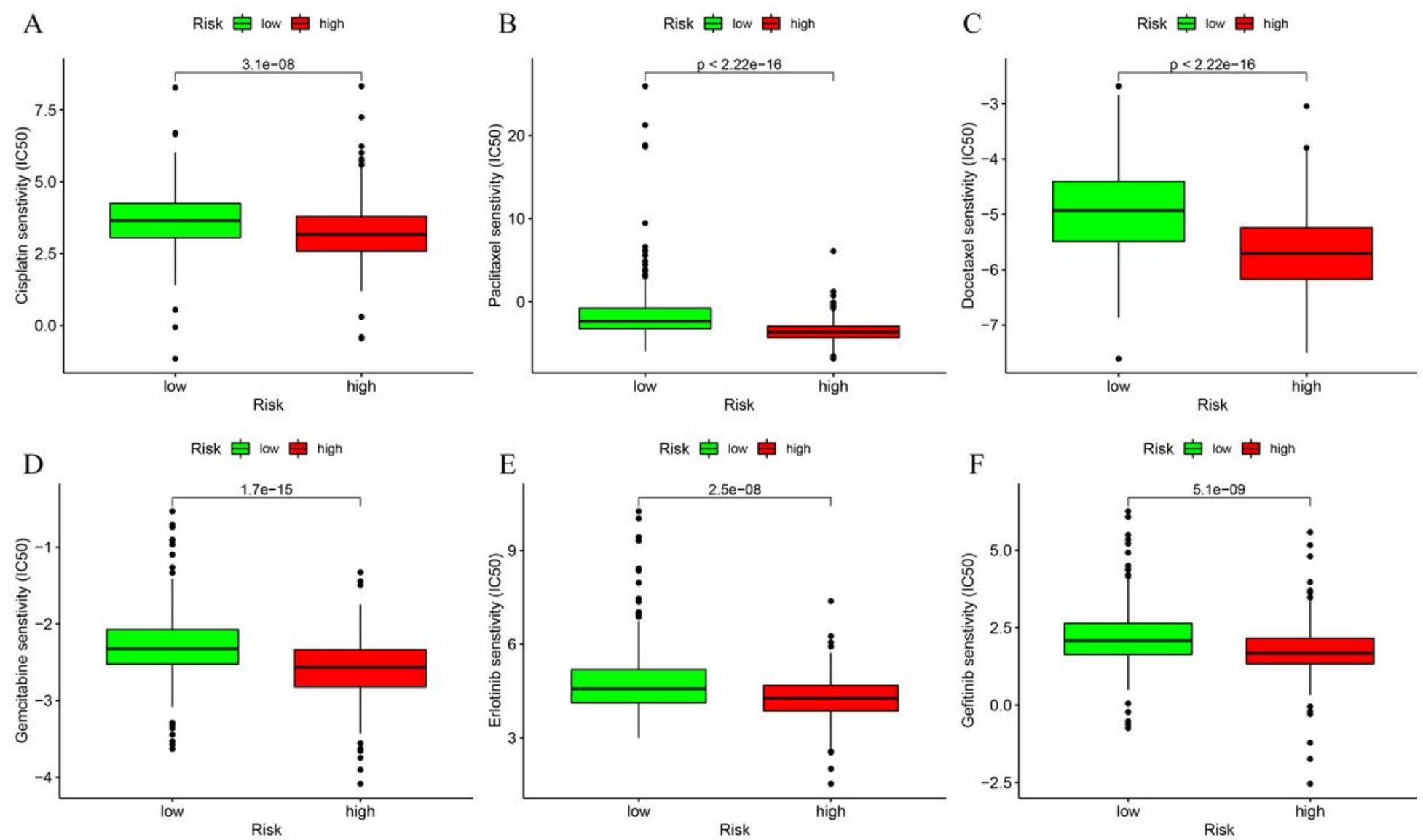

Figure 10

The risk model was used to evaluate IC50 differences. Analysis of (A) cisplatin, (B) paclitaxel, (C) docetaxel, (D) gemcitabine, (E) erlotinib, and (F) gefitinib showed that there was significant difference between the low- and high-risk groups.

\section{Supplementary Files}

This is a list of supplementary files associated with this preprint. Click to download.

- Supplementaryfile1.xls

- Supplementaryfile2.pdf 